\title{
Talaporfin-mediated photodynamic therapy for peritoneal metastasis of gastric cancer in an in vivo mouse model: Drug distribution and efficacy studies
}

\author{
KENTARO KISHI $^{1}$, MASAHIKO YANO ${ }^{1}$, MASAHIRO INOUE ${ }^{2}$, ISAO MIYASHIRO ${ }^{1}$, MASAAKI MOTOORI ${ }^{1}$, \\ KOJI TANAKA ${ }^{1}$, KUNIHITO GOTO ${ }^{1}$, HIDETOSHI EGUCHI ${ }^{1}$, SHINGO NOURA ${ }^{1}$, TERUMASA YAMADA ${ }^{1}$, \\ MASAYUKI OHUE ${ }^{1}$, HIROAKI OHIGASHI ${ }^{1}$ and OSAMU ISHIKAWA ${ }^{1}$
}

Departments of ${ }^{1}$ Surgery and ${ }^{2}$ Biochemistry, Osaka Medical Center for

Cancer and Cardiovascular Diseases, Osaka, Japan

Received August 20, 2009; Accepted October 16, 2009

DOI: 10.3892/ijo_00000502

\begin{abstract}
Photodynamic therapy (PDT) is a potential treatment for the peritoneal dissemination of gastric cancer, because its cytotoxicity is limited to superficial lesions. We examined the accumulation of talaporfin in peritoneal metastatic nodules and determined the optimal laser condition for these nodules. We also evaluated the pathological response after therapy. We created a peritoneal metastasis model in nude mice using the MKN-45 EGFP cell line. We evaluated the accumulation of talaporfin in peritoneal metastatic nodules and normal organs by spectrophotometric analysis $2-8 \mathrm{~h}$ after i.p. talaporfin. To determine optimal PDT conditions, we treated metastatic nodules and the small intestine using multiple laser doses $\left(2,5\right.$, and $10 \mathrm{~J} / \mathrm{cm}^{2}$, respectively). Accumulation of talaporfin was detected in metastatic nodules in higher intensities than in the small intestine. The fluorescent intensity of the peritoneal metastatic nodules gradually decreased dependent on the time interval between the laser treatment and talaporfin administration. Fluorescent intensity in the small intestine decreased more than in the metastatic nodules. The pathological response rates by dose were $52.5 \%$ at $2 \mathrm{~J} / \mathrm{cm}^{2}$, $43.2 \%$ at $5 \mathrm{~J} / \mathrm{cm}^{2}$, and $64.4 \%$ at $10 \mathrm{~J} / \mathrm{cm}^{2}$, respectively, when the laser treatment was used $2 \mathrm{~h}$ after talaporfin administration, whereas at $4 \mathrm{~h}$, they were $20.8,25.5$, and $26.2 \%$, respectively. Finally, the recommended treatment conditions were considered to be a $2 \mathrm{~J} / \mathrm{cm}^{2}$ laser dose and a 4-h interval in terms of toxicity. Talaporfin-mediated PDT may be an effective treatment modality for patients with advanced gastric adenocarcinoma and metastatic peritoneal nodules.
\end{abstract}

Correspondence to: Dr Kentaro Kishi, Department of Surgery, Osaka Medical Center for Cancer and Cardiovascular Diseases, 1-3-3 Nakamichi, Higashinariku, Osaka, Osaka 537-8511, Japan E-mail: kisi-ke@mc.pref.osaka.jp

Key words: talaporfin, PDT, peritoneal metastasis, gastric cancer

\section{Introduction}

Gastric cancer remains a worldwide health problem, accounting for $10 \%$ of all new cancer diagnoses and $12 \%$ of all cancerrelated deaths. Diagnosis of gastric cancer is often made when the disease is advanced and unresectable (1). In Japan, a nation-wide screening program has resulted in early diagnosis and prompt surgical intervention and has thus improved the prognosis of patients with primary gastric cancer, resulting in a 5-year over all survival rate of $68.2 \%$ (2). However, the prognosis of patients with advanced gastric cancer remains poor, with 5-year survival rates of $43.5 \%$ for stage III cancer and $9.9 \%$ for stage IV cancer.

Peritoneal dissemination is one of the most common recurrence patterns after radical resection of advanced gastric cancer; it is observed in $25.6 \%$ of patients with serosa-positive gastric cancer (2). Peritoneal dissemination is thought to be caused by micrometastatic nodules on peritoneal surfaces or floating cancer cells in the abdominal cavity that were not detected at the time of surgery. If peritoneal recurrence rates can be reduced, the prognosis of advanced gastric cancer will be dramatically improved.

To date, many clinical trials aimed at decreasing peritoneal recurrences have been conducted. These trials have studied such treatment modalities as systemic adjuvant chemotherapy (3-8), intraperitoneal chemoperfusion with or without hyperthermia (9) and chemoradiotherapy $(10,11)$. Although several meta-analyses showed a marginally significant benefit of some of the above therapies, no single randomized clinical trial has demonstrated a significant survival benefit. It is therefore widely thought that there is currently no standard effective therapy for preventing peritoneal recurrence of gastric cancer.

In photodynamic therapy (PDT), a systemically administered photosensitizing agent is activated by laser light of a specific wavelength delivered by an optical fiber. Lightactivated photosensitizer molecules react with endogenous oxygen, resulting in the generation of singlet oxygen, which initiates a series of intracellular events that result in the destruction of target tissues while avoiding significant side effects in the patient. With respect to side-effect profiles 
and damage to normal tissue, PDT has the advantage over radiation because the photosensitizing agent used in PDT specifically accumulates in cancer cells as opposed to normal cells. PDT has become increasingly accepted as a treatment option for early lung cancers, gastric cancer, esophageal cancer, and others (12-14), and has also been clinically applied in poor surgical candidates with advanced cancers (15-17). Because laser energy penetrates to a depth of only $2 \mathrm{~mm}$ in tissues $(18,19)$, the cytotoxicity of PDT is limited to the treatment of superficial lesions. Therefore, disseminated small nodules on the peritoneal surface could potentially be suitable targets for PDT.

Talaporfin, a second-generation photosensitizer, has several advantages over the first-generation photosensitizers, including a lack of prolonged photosensitization and a shorter interval (4-6 h) required between drug administration and laser light exposure $(20,21)$ as compared to photofirin, a firstgeneration drug that requires 48-72 h. Talaporfin-mediated PDT has been examined in the treatment of several solid tumors $(22,23)$. However, thus far, no experimental or clinical studies have used talaporfin-mediated PDT for the treatment of peritoneally disseminated gastric cancer. In this study, we examined the efficacy of talaporfin-mediated PDT in the treatment of peritoneal gastric cancer micrometastases using a mouse model.

\section{Materials and methods}

Cell culture. MKN-28 and MKN-45 EGFP (which was made by transfecting pEGFP-N1 into the MKN-45 cell line) cells were provided by the Department of Gastroenterological Surgery, Graduate School of Medicine, Osaka University, Osaka, Japan (24). MKN-7 cells were purchased from the Health Science Research Resources Bank (Osaka, Japan). The cells were cultured in RPMI-1640 medium with 10\% FBS and antibiotics.

Photosensitizer and laser light delivery system. We used talaporfin sodium provided by Meiji Seika Kaisha Ltd., Tokyo, Japan. It was reconstituted in normal saline to a final concentration of $25 \mathrm{mg} / \mathrm{ml}$ and stored in the dark at $-20^{\circ} \mathrm{C}$ until used. The laser we used was a diode laser system (Diode laser, Panasonic Shikoku Electronics Co., Ltd., Yokohama, Japan). The treatment wavelengths used were $664 \mathrm{~nm}$ and $150 \mathrm{~mW} / \mathrm{cm}^{2}$.

Spectrophotometric analysis device. We examined the uptake and accumulation of talaporfin in peritoneal metastatic nodules, liver and small intestine using a semiconductor laser with a VLD-M1 spectrometer (M\&M Co., Ltd., Tokyo, Japan) that exposed a laser light with a peak wavelength of $405 \pm 1 \mathrm{~nm}$ and a light output of $140 \mathrm{~mW}$. The spectrometer and its accessory software (BW-Spec V3.24; B\&W TEK, Inc., Newark, Del., USA) were used to analyze the spectrum waveform (25) and revealed an amplitude peak (relative fluorescent intensity) at a wavelength of $508 \mathrm{~nm}$ for MKN-45 EGFP, at $505 \mathrm{~nm}$ for autofluorescence and at $678 \mathrm{~nm}$ for talaporfin. The relative intensity of the talaporfin solution (with concentrations ranging from 0 to $50 \mu \mathrm{g} / \mathrm{ml}$ ), which was measured by the spectrometer, was observed to have a linear correlation with the talaporfin concentration. To reduce measurement error, we compared the relative fluorescent intensity ratio of talaporfin in the target tissue, which was calculated by dividing the relative fluorescent intensity by that of GFP, or the known autofluorescence.

An in vivo mouse model of peritoneal metastasis. Five-weekold BALB/c male nude mice (CLEA Japan Inc., Osaka, Japan) were used in this study. We created a peritoneal dissemination model using the method described below. An aliquot of $1 \times 10^{6} \mathrm{MKN}-45$ EGFP cells was injected into the peritoneal cavity of nude mice. Seven days after injection of the cells, mice were subjected to fluorescent stereomicroscopic observation, and several GFP fluorescent nodules, which were $<1 \mathrm{~mm}$ in diameter, were detected on the omentum and mesenterium. The animal experiments were carried out according to the Institutional ethics guidelines.

Cytotoxicity of talaporfin-mediated PDT on cell lines. The cytotoxic effect of PDT was measured using the CellTiter 96 Aqueous One Solution cell proliferation assay (Promega, Madison, WI). MKN-45 EGFP cells were suspended in RPMI-1640 with $10 \%$ FBS and subsequently placed into 96-well plates at a concentration of $2 \times 10^{3}$ cells/well. MKN-7 and MKN-28 cells were plated at a concentration of $4 \times 10^{3}$ cells/well. The cells were incubated at $37^{\circ} \mathrm{C}$ for $24 \mathrm{~h}$. The indicated concentration of talaporfin $(0-30 \mu \mathrm{g} / \mathrm{ml})$ was then added and cells were incubated for another $24 \mathrm{~h}$. After incubation, the cells were washed twice with PBS(-), resuspended in fresh medium, and treated with laser light at one of the following doses: $2,5,10,30$, or $50 \mathrm{~J} / \mathrm{cm}^{2}$. After the laser treatment, the cells were incubated for $48 \mathrm{~h}$ at $37^{\circ} \mathrm{C}$. The CellTiter 96 Aqueous One Solution was then added to the wells, and the absorbency was measured $3 \mathrm{~h}$ later at a wavelength of $490 \mathrm{~nm}$ on an automatic microplate reader (Benchmark; Bio-Rad, Hercules, CA). The survival ratio of the treated cells was compared to that of untreated control cells that did not receive PDT.

Evaluation of talaporfin concentration in an in vivo mouse model of peritoneal metastasis. Talaporfin sodium (5 or $10 \mathrm{mg} /$ $\mathrm{kg}$ ) was administered intraperitoneally to the peritoneal dissemination model mice 14 days after initial cell injection. The mice were laparotomized at 2, 4 and $8 \mathrm{~h}$ after talaporfin administration, and peritoneal metastatic nodules were excised and washed with normal saline. Next, we measured the relative fluorescent intensity ratio of talaporfin using a spectrometer. We also measured the same ratio in liver and intestine using the same method at 2, 4 and $8 \mathrm{~h}$ after intraperitoneal administration of $10 \mathrm{mg} / \mathrm{kg}$ of talaporfin.

Evaluation of the pathological response of PDT. We treated the target nodules using 2, 5, and $10 \mathrm{~J} / \mathrm{cm}^{2}$ doses of laser light using a special probe that adjusted the photo-radiated field to $5 \mathrm{~mm}$ in diameter and shaded the region outside of the target to minimize damage to normal tissues. We then performed a second laparotomy on PDT day 3, excised the tissue in the treatment field, and preserved it in formalin.

The removed tissues were fixed in $10 \%$ formaldehyde and sliced into $0.5 \mathrm{~mm}$ serial longitudinal sections. The pathological 

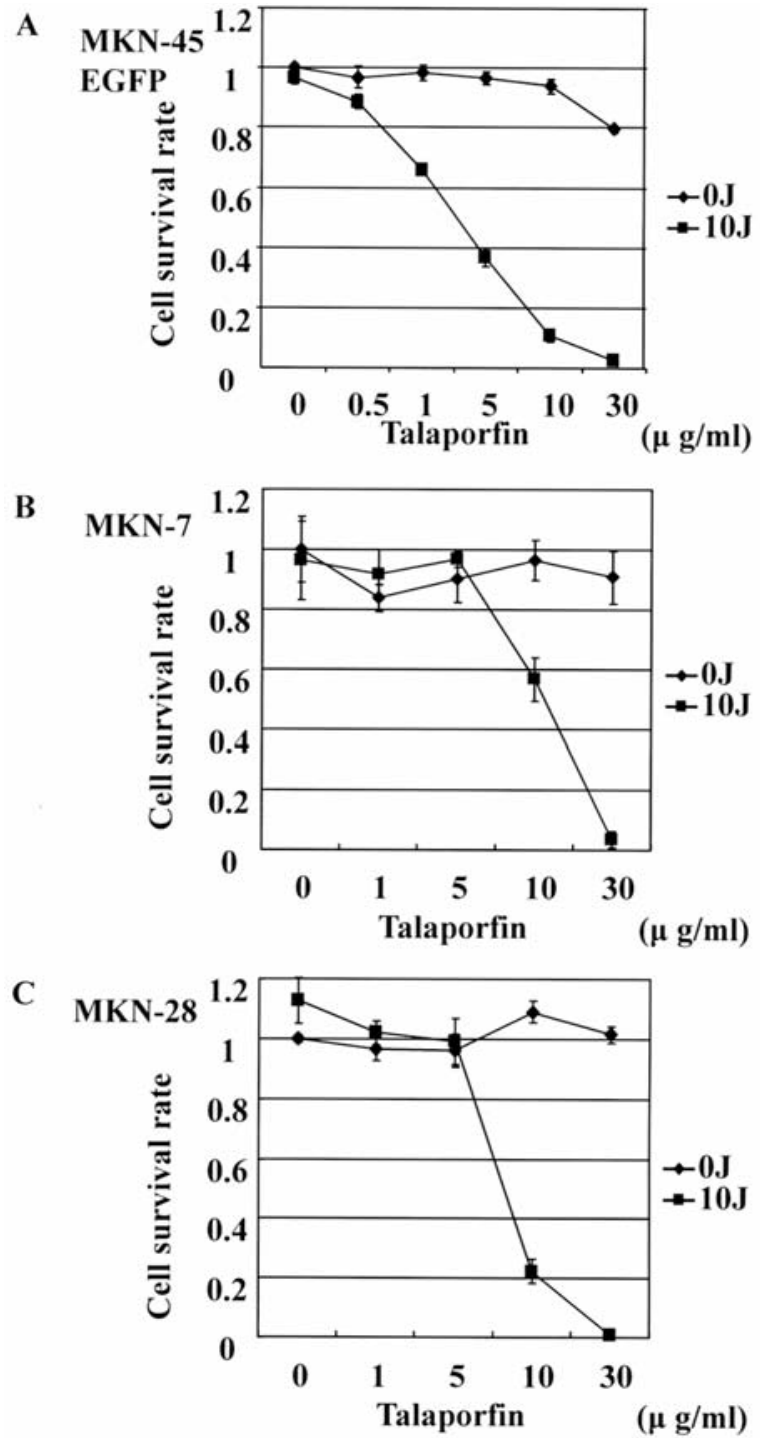

Figure 1. The gastric cancer cell lines (A) MKN-45 EGFP, (B) MKN-7, and (C) $\mathrm{MKN}-28$ were incubated with a variable concentration of talaporfin (range 0-30 $\mu \mathrm{g} / \mathrm{ml}$ ) for $24 \mathrm{~h}$, and were then treated with laser light at a dose of $0 \mathrm{~J} / \mathrm{cm}^{2}$ (controls) or $10 \mathrm{~J} / \mathrm{cm}^{2}$ (cases). In order to cause cell death in $>50 \%$ cell deaths using this laser dose, at least 5 and $10 \mu \mathrm{g} / \mathrm{ml}$ of talaporfin were necessary for MKN-45 EGFP cells (A) and for MKN-7 (B) and MKN-28 cells $(\mathrm{C})$, respectively.

response of PDT was evaluated by comparing the proportion of viable cancer cells with that of all cancer cells in the tissue in hematoxylin and eosin-stained sections of the surgical specimens. Pathologic response was classified as follows: grade 1 , a minor effect, with viable cancer cells accounting for more than two-thirds of the tumor tissue; grade 2, a moderate effect, with viable cancer cells accounting for more than one-third but less than two-thirds of the tumor tissue; and grade 3, a pathological response in which viable cancer cells account for less than one-third of the tumor tissue. Grade 3 also includes specimens that have no residual viable cancer cells.

Statistical analysis. Associations between the pathological effects in two groups were analyzed using the Fisher's exact test. In all analyses, P-values $<0.05$ were considered statistically significant. All statistical analyses were performed using the

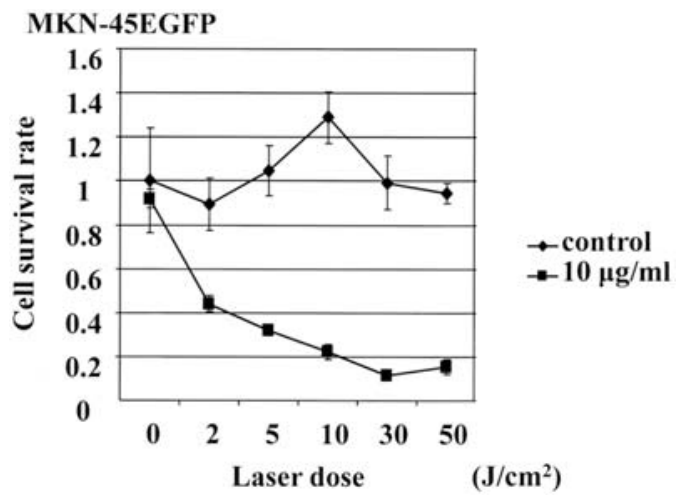

Figure 2. After MKN-45 EGFP cells were incubated with 10 (treatment group) or $0 \mu \mathrm{g} / \mathrm{ml}$ (control group) of talaporfin for $24 \mathrm{~h}$, they were treated with the indicated dose of laser light $\left(0-50 \mathrm{~J} / \mathrm{cm}^{2}\right)$. More than $50 \%$ cell death was obtained at a dose of $2 \mathrm{~J} / \mathrm{cm}^{2}$, and the survival rate of MKN-45 EGFP cells gradually decreased and reached a plateau at a dose of $30 \mathrm{~J} / \mathrm{cm}^{2}$.

StatView software package, version 5.0 (Abacus Concepts, Inc., Berkeley, CA).

\section{Results}

The effects of PDT on cultured cancer cell lines. The in vitro anti-cancer effects of talaporfin-mediated PDT were evaluated using three gastric cancer cell lines: MKN-45 EGFP, MKN-7 and MKN-28. After each cell line was incubated with one of the indicated concentrations of talaporfin $(0-30 \mu \mathrm{g} / \mathrm{ml})$ for $24 \mathrm{~h}$, they were treated with laser light at a dose of $10 \mathrm{~J} / \mathrm{cm}^{2}$. The cell survival rate was estimated by the MTS assay. Talaporfin showed strong anti-tumor effects in all three cell lines in a dose-dependent manner in cells that were treated with PDT, whereas in the control cell lines that did not undergo PDT, talaporfin did not have any significant anti-tumor effects. To obtain a cell death rate of $>50 \%$ using a $10 \mathrm{~J} / \mathrm{cm}^{2}$ dose, at least $5 \mu \mathrm{g} / \mathrm{ml}$ of talaporfin was necessary in MKN-45 EGFP cells (Fig. 1A) and $10 \mu \mathrm{g} / \mathrm{ml}$ of talaporfin was necessary for MKN-7 (Fig. 1B) and MKN-28 cells (Fig. 1C). Next, we examined the dose-dependent effects of laser energy on survival of MKN-45 EGFP cells. After MKN-45 EGFP cells were incubated with $10 \mu \mathrm{g} / \mathrm{ml}$ of talaporfin for $24 \mathrm{~h}$, they were treated with the indicated dose of laser light $\left(0-50 \mathrm{~J} / \mathrm{cm}^{2}\right)$. A cell death rate higher than $50 \%$ was obtained at a $2 \mathrm{~J} / \mathrm{cm}^{2}$ dose, and the survival rate of MKN-45 EGFP cells gradually decreased, reaching a plateau at $30 \mathrm{~J} / \mathrm{cm}^{2}$ (Fig. 2).

Uptake and accumulation of talaporfin in peritoneal metastatic nodules. Before starting in vivo experiments, we first examined whether talaporfin accumulated in peritoneal metastatic nodules. Fourteen days after the i.p. injection of $1 \times 10^{6}$ of MKN-45 EGFP cells into the abdominal cavity of mice (by which time the peritoneal metastases should reach 1-2 mm in diameter according to our preliminary studies), $10 \mathrm{mg} / \mathrm{kg}$ of talaporfin was intraperitoneally administered. The mice were sacrificed 2, 4 and $8 \mathrm{~h}$ later, and their micrometastatic nodules (tagged with GFP) were excised and subjected to spectrometric analysis (VLD-M1 M\&M Co. Ltd., Osaka, Japan) to determine the talaporfin concentration in the metastatic foci. Samples of liver and small intestine were 

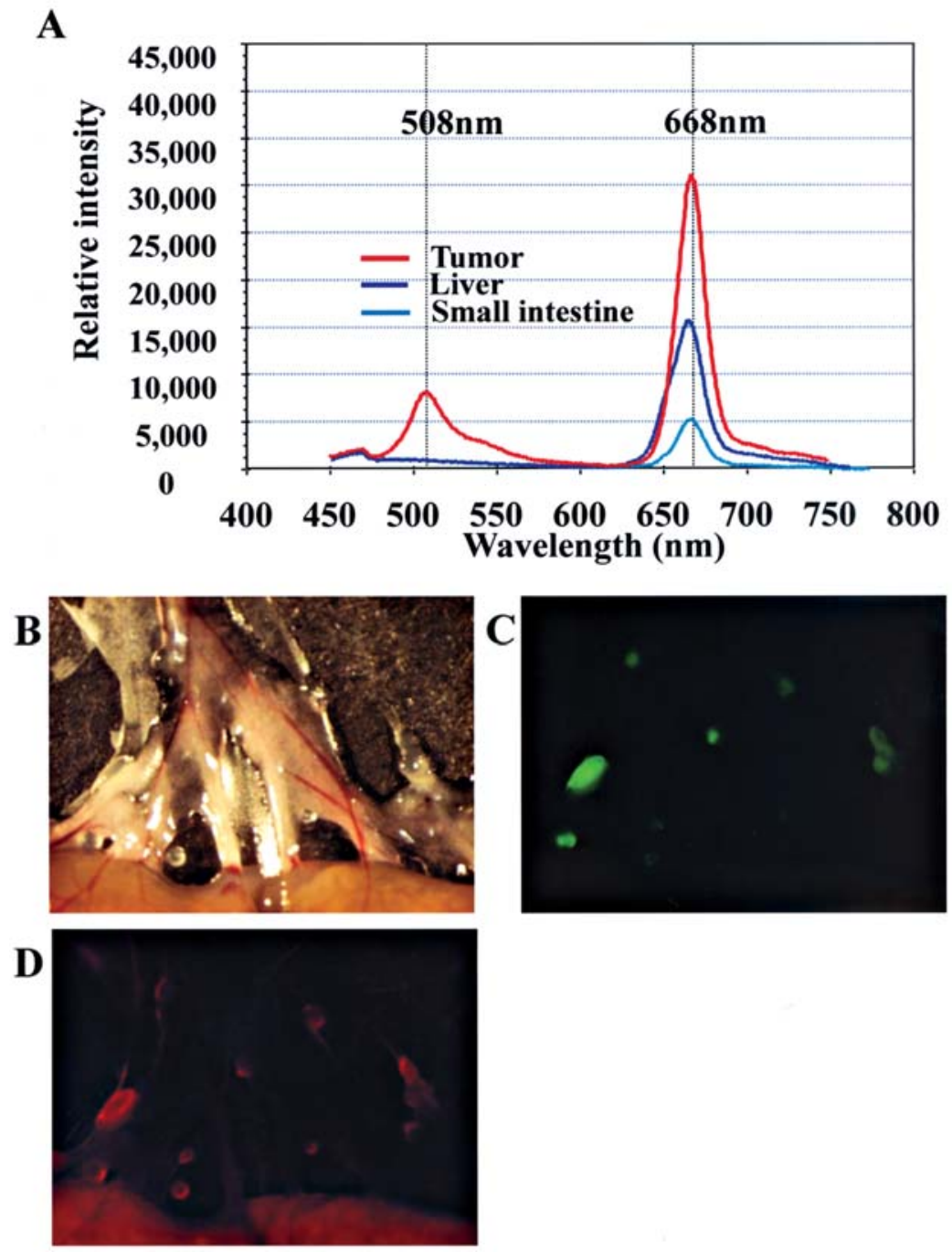

Figure 3. (A) Ten $\mathrm{mg} / \mathrm{kg}$ of talaporfin was intraperitoneally administered to mice that had been inoculated with MKN-45 EGFP to create an in vivo model of peritoneal metastasis. Four hours after talaporfin administration, the spectrum waveform of the peritoneal metastatic nodules, liver and small intestine were analyzed by a VLD-M1 spectrometer. Peritoneal metastatic nodules showed two peaks of fluorescence emission spectra by spectrophotometric analysis, one at $508 \mathrm{~nm}$, corresponding to GFP, and another at $668 \mathrm{~nm}$, corresponding to talaporfin. The small intestine also showed a weak but significant level of fluorescence at $668 \mathrm{~nm}$. Fluoromicroscopic findings of the nodules in mice that were intraperitoneally injected with MKN-45 EGFP cells followed by $10 \mathrm{mg} / \mathrm{kg}$ of i.p. talaporfin. The peritoneal micrometastatic nodules were observed by normal white light microscopy (B). Green fluorescence (indicating GFP) (C), and red fluorescence (indicating talaporfin) (D) were observed using fluorescence microscopy.

also removed and subjected to spectrophotometric analysis.

Peritoneal metastatic nodules showed two peaks of fluorescence emission spectra by spectrophotometric analysis: one at $508 \mathrm{~nm}$, corresponding to GFP, and another at $678 \mathrm{~nm}$, corresponding to talaporfin, while liver and small intestine also showed a weak but notable level of fluorescence at $678 \mathrm{~nm}$ (Fig. 3A). Fig. 3B shows the fluoromicroscopic findings of the peritoneum of mice that were intraperitoneally injected with MKN-45 EGFP cells and subsequently treated with talaporfin. Micrometastatic nodules on the peritoneal surface had a red fluorescence pattern and were easily detectable by fluoromicroscopy, suggesting that the talaporfin had accumulated in the cancer cells. Furthermore, we also examined the uptake and accumulation of intravenously injected talaporfin into peritoneal metastatic nodules. Compared with i.p. administration, intravenous (i.v.) administration of talaporfin resulted in less fluorescent intensity in the metastatic nodules as analyzed by spectrometry (Fig. 4B) and poorer visualization by fluoromicroscopy (data not shown). Therefore, for the subsequent experiments, talaporfin was administered via an i.p. route.

Optimal dose of injected talaporfin and optimal time-point of laser treatment in an in vivo mouse model of peritoneal metastasis. To determine the optimal talaporfin dose, we measured the relative fluorescence intensity ratio in peritoneal metastatic nodules after i.p. administration of various doses of talaporfin (Fig. 4A). The relative fluorescent intensity ratio of metastastic nodules was calculated by dividing the relative fluorescent intensity of these tissues by that of GFP. Mice treated with $10 \mathrm{mg} / \mathrm{kg}$ of talaporfin had a relative intensity ratio that was about 3 -fold higher than that in mice treated with $5 \mathrm{mg} / \mathrm{kg}$ of talaporfin. Next, to determine the optimal time-point of laser treatment after talaporfin administration at which cytotoxic effects on normal tissues are reduced while cytotoxic effects on tumor cells are maintained - we 


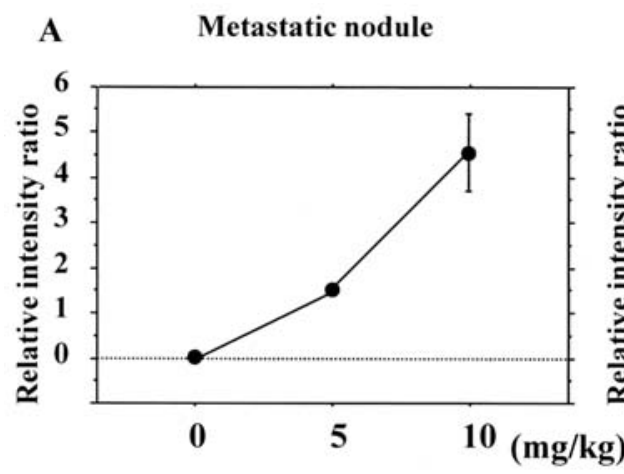

B Metastatic nodule

Talaporfin concentration

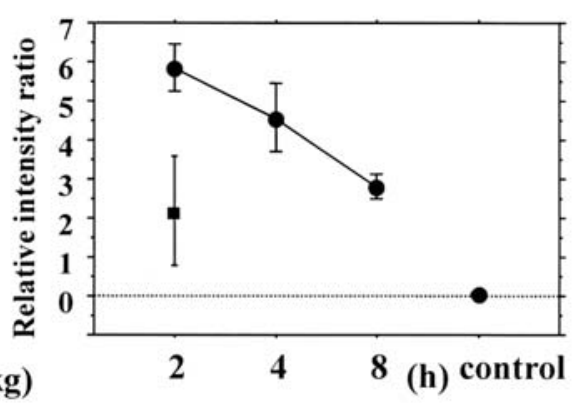

Time after talaporfin administaration

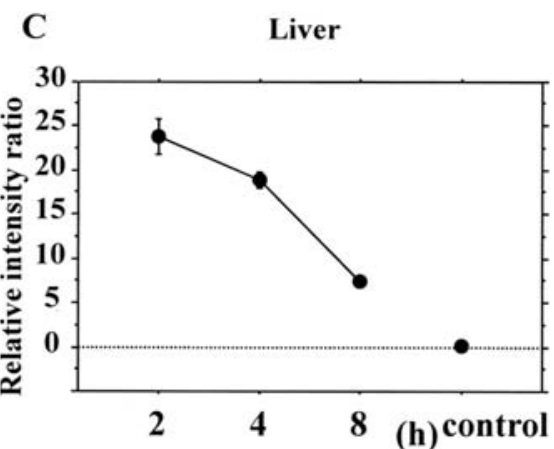

D Small intestine

Time after talaporfin administaration

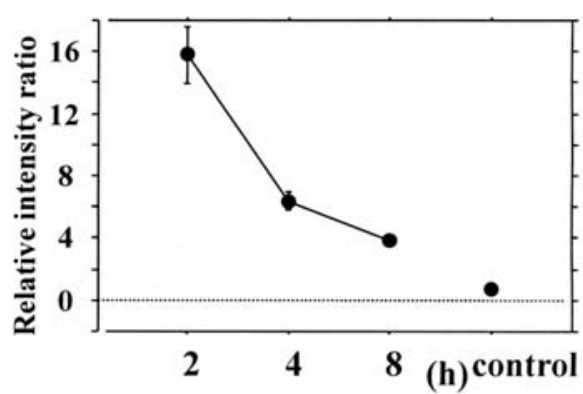

Time after talaporfin administaration

Figure 4. (A) Four hours after intraperitoneal injection of talaporfin, the mice treated with a $10 \mathrm{mg} / \mathrm{kg}$ dose of talaporfin showed a relative intensity ratio that was about 3-fold higher than that observed in mice treated with a $5 \mathrm{mg} / \mathrm{kg}$ dose (B). After intraperitoneal injection of $10 \mathrm{mg} / \mathrm{kg}$ of talaporfin, the relative fluorescent intensity ratio in the peritoneal tumors gradually decreased over time compared to the ratio observed at $2 \mathrm{~h}$, with $78 \%$ present at $4 \mathrm{~h}$ and $48 \%$ present at $8 \mathrm{~h}(\bullet)$. The relative fluorescent intensity ratio $2 \mathrm{~h}$ after intravenous administration ( $\mathbf{a})$ of talaporfin was lower than that obtained via intraperitoneal injection. Relative fluorescent ratios in liver (C) and small intestine (D) were decreased (as compared to the $2 \mathrm{~h}$ value) to $79 \%$ of the $2 \mathrm{~h}$ value at $4 \mathrm{~h}, 31 \%$ at $8 \mathrm{~h}, 36 \%$ at $4 \mathrm{~h}$ and $24 \%$ at $8 \mathrm{~h}$, respectively.

measured talaporfin concentration over time in mouse peritoneal metastatic nodules, liver and small intestines using spectrometry. Relative fluorescent intensity ratios of the liver and the small intestine were calculated by dividing the relative fluorescent intensity of these tissues by the known autofluorescent intensity. The relative fluorescent intensity ratios in peritoneal tumors gradually decreased with time as compared with the relative fluorescent intensity ratio present $2 \mathrm{~h}$ after talaporfin administration (78\% was present at $4 \mathrm{~h}$ and $48 \%$ was present at 8 h) (Fig. 4B). In liver and small intestine, relative fluorescent intensity ratios were decreased to $79 \%$ at $4 \mathrm{~h}$ and $31 \%$ at $8 \mathrm{~h}$, and $36 \%$ at $4 \mathrm{~h}$ and $24 \%$ at $8 \mathrm{~h}$, respectively, as compared with the ratio at $2 \mathrm{~h}$ (Fig. $4 \mathrm{C}$ and D).

Pathological response in peritoneal cancer nodules by PDT. We evaluated the effects of talaporfin-mediated PDT on peritoneal metastases using an in vivo mouse model of peritoneal metastasis. Seven days after i.p. injection of $1 \times 10^{6}$ of MKN-45 EGFP cells, mice were given $10 \mathrm{mg} / \mathrm{kg}$ of talaporfin intraperitoneally. Mice were laparotomized $2 \mathrm{~h}$ or $4 \mathrm{~h}$ later, and then treated with varying doses of laser light.

Twenty-four hours after the laser treatment, the mice were re-laparotomized to assess the effects of PDT. We found edematous changes and few or no adhesions on the laser-treated tissues macroscopically. However, a white color change was frequently noted on the liver, which was found to be coagulative necrosis on microscopic analysis. The average size of targeted omental nodules was $696 \pm 474 \mu \mathrm{m}$.

In mice that underwent PDT $2 \mathrm{~h}$ after talaporfin administration, a grade 3 response was seen in $52.5 \%$ of cancer nodules at a laser dose of $2 \mathrm{~J} / \mathrm{cm}^{2}$, in $43.2 \%$ at a dose of $5 \mathrm{~J} / \mathrm{cm}^{2}$, and in $64.4 \%$ at a dose of $10 \mathrm{~J} / \mathrm{cm}^{2}$, respectively. In mice that underwent PDT $4 \mathrm{~h}$ after talaporfin administration, the rates of a grade 3 response were $20.8 \%$ at a dose of $2 \mathrm{~J} / \mathrm{cm}^{2}$, $25.5 \%$ at a dose of $5 \mathrm{~J} / \mathrm{cm}^{2}$, and $26.2 \%$ at a dose of $10 \mathrm{~J} / \mathrm{cm}^{2}$, respectively. Anti-tumor effects were significantly dependent on the time interval between the laser treatment and talaporfin administration at all laser doses $\left(2 \mathrm{~J} / \mathrm{cm}^{2}, \mathrm{P}<0.0001 ; 5 \mathrm{~J} / \mathrm{cm}^{2}\right.$ : $\mathrm{P}=0.022 ; 10 \mathrm{~J} / \mathrm{cm}^{2}, \mathrm{P}<0.0001$ ), but it was independent of the laser dose at all time intervals (Fig. 5).

To determine PDT conditions that would be tolerable in terms of toxicity, we treated the small intestine and mesenterium 1-6 cm proximal to the ileocecal junction with various laser doses. In mice that were treated with a $2 \mathrm{~h}$ interval between talaporfin administration and laser treatment, substantial edematous changes were seen in the ileum at a dose of $2 \mathrm{~J} / \mathrm{cm}^{2}$, and remarkable edematous and ischemic changes were seen at doses of 5 and $10 \mathrm{~J} / \mathrm{cm}^{2} 1$ day after PDT. The mice that were treated with a laser dose of $2 \mathrm{~J} / \mathrm{cm}^{2}$ with a $2 \mathrm{~h}$ interval $(\mathrm{n}=5)$ all died of intestinal perforation 


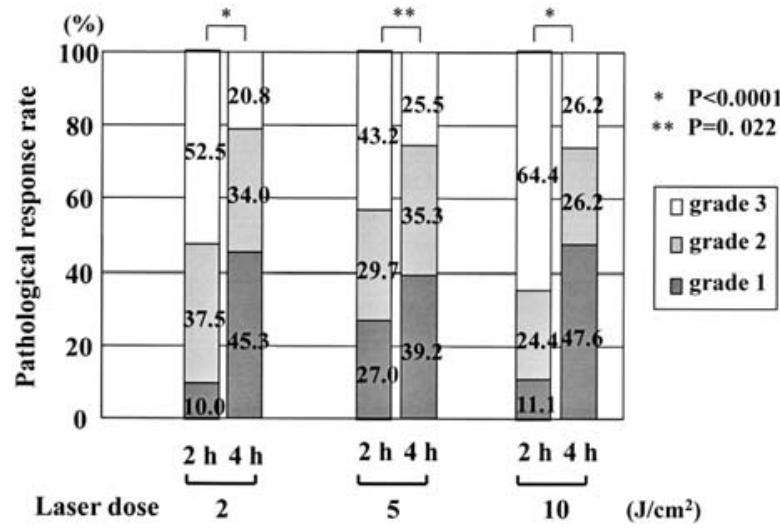

Figure 5. Anti-tumor pathological effects were significantly dependent on the time interval between laser treatment and talaporfin administration at all laser doses $\left(2 \mathrm{~J} / \mathrm{cm}^{2}, \mathrm{P}<0.0001 ; 5 \mathrm{~J} / \mathrm{cm}^{2}, \mathrm{P}=0.022 ; 10 \mathrm{~J} / \mathrm{cm}^{2}, \mathrm{P}<0.0001\right)$, but they were independent of the laser dose at both times.

within 3 days after PDT treatment. On the other hand, in mice treated with a $4 \mathrm{~h}$ interval, remarkable edema was noted at a dose of $10 \mathrm{~J} / \mathrm{cm}^{2}$, but only slight edematous changes were noted at a dose of $5 \mathrm{~J} / \mathrm{cm}^{2}$ and no changes were noted at a dose of $2 \mathrm{~J} / \mathrm{cm}^{2}$. All the mice that were treated with a laser dose of $2 \mathrm{~J} / \mathrm{cm}^{2}$ with a $4 \mathrm{~h}$ interval $(\mathrm{n}=5)$ survived without any complications until 30 days after PDT treatment. Thus, the optimal treatment conditions for talaporfin-mediated PDT are considered to be a laser dose of $2 \mathrm{~J} / \mathrm{cm}^{2}$ and a $4 \mathrm{~h}$ interval between talaporfin administration and laser treatment (Table I).

\section{Discussion}

PDT has proved to be a promising new therapeutic modality in the treatment of cancer. Porphyrin-based photosensitizers such as hematoporphyrin derivative and porfimer sodium are most often used clinically for the treatment of cancer. But recently, promising results using new generation photosensitizers, such as talaporfin and 5-aminolevulinic acids (ALA), are frequently reported to shorten the in vivo retention that increases the risk of phototoxicity and the interval required between drug administration and laser treatment. This study is the first to show that intraperitoneally injected talaporfin accumulates in peritoneal metastatic nodules from gastric cancer and that talaporfin-mediated PDT exerts substantial anti-tumor effect in vivo (using a mouse model of peritoneal metastasis).

With regard to 5-aminolevulinic acids (ALA), numerous studies have reported that 5-ALA is useful for photodynamic diagnosis (PDD) of various malignant tumors (26-28). Several reports have shown promising preliminary results of 5-ALAmediated photodynamic therapy (PDT) in the treatment of malignant tumors in the abdominal cavity $(29,30)$. However, there are no reports examining 5-ALA-mediated PDT for peritoneal metastasis in gastrointestinal cancers, probably because of its low anti-tumor effects $(31,32)$.

On the other hand, porphyrin-mediated PDT was reported to show survival benefit in animal models of peritoneal metastasis. Also, clinical trials examining debulking surgery followed by intraoperative photofirin-mediated PDT for peritoneal or pleural dissemination showed good local control rates with acceptable toxicity (33-35). The anti-tumor effects of photofirin-mediated PDT were more potent than those of 5-ALA-mediated PDT, but the toxicity rates were higher. Surgical debulking and i.p. PDT using photofirin for peritoneal dissemination was associated with a risk of capillary leak syndrome, which is a significant inflammatory response syndrome after surgery and necessitates massive fluid resuscitation, careful ICU monitoring, and, frequently, prolonged ventilator support (36). Capillary leak syndrome is essentially considered to be burn of an extensive surface area of the peritoneal cavity. If PDT is to become a clinically useful therapy, it must prevent the occurrence of capillary leak syndrome.

In our study examining talaporfin-mediated PDT, no lethal side effects, such as capillary leak syndrome, were noted. Talaporfin-mediated PDT has the advantage of a lack of prolonged photosensitization because the drug washes out early in normal tissue. Talaporfin-mediated PDT may therefore be less toxic than photofirin-mediated PDT and may reduce the risk of capillary leak syndrome, although these findings need to be confirmed in clinical trials.

Initially, we employed an i.v. route for talaporfin administration because this method had been used previously in mouse experiments $(18,37)$. However, our preliminary study showed that, as compared with i.v. administration, i.p. administration resulted in a higher accumulation of talaporfin in the metastatic nodules as well as a more complete pathological effect (data not shown).

Gormer et al (20) showed that talaporfin concentration in subcutaneous tumors and other organs did not significantly differ according to the mode of drug administration (i.v. versus i.p), the tumor size or the time of laser treatment ( $4 \mathrm{~h}$ and $24 \mathrm{~h}$ after talaporfin administration). The difference between Gormer's results and ours may be explained by the difference of targeted tumor sites (subcutaneous tumors vs. peritoneal tumors). Particularly in the case of peritoneal

Table I. Toxicity: optical findings of the ileum 1 day after PDT and mortality.

Interval between drug injection and laser treatment
Laser dose $\left(\mathrm{J} / \mathrm{cm}^{2}\right)$

\begin{tabular}{|c|c|c|c|c|}
\hline \multirow{2}{*}{$\begin{array}{l}\text { Interval between drug } \\
\text { injection and laser treatment }\end{array}$} & \multicolumn{3}{|c|}{ - } & \multirow{2}{*}{$\begin{array}{l}\text { Died/number of mice (day after } \\
\text { treated at a laser dose of } 2 \mathrm{~J} / \mathrm{cm}^{2} \text { ) }\end{array}$} \\
\hline & 2 & 5 & 10 & \\
\hline $2 \mathrm{~h}$ & $(+)$ & $(++)^{\mathrm{a}}$ & $(++)^{\mathrm{a}}$ & $5 / 5$ (2 at day 2,3 at day 3$)$ \\
\hline $4 \mathrm{~h}$ & $(-)$ & $(+)$ & $(++)$ & $0 / 5$ \\
\hline
\end{tabular}


metastatic nodules, which have poor tumor blood supply, cancer cells seem to be more directly exposed to intraperitoneally administered talaporfin than to intravenously administered talaporfin. As shown in this study, the relative intensity ratio of peritoneal tumors $2 \mathrm{~h}$ after talaporfin administration was higher in animals administered talaporfin by the i.p. route than in those treated with i.v. talaporfin (Fig. 4B). In the photofirin study, Perry et al showed no difference in the intestinal uptake of photofirin according to the route of administration, but did demonstrate an increased photofirin elimination half-time in peritoneal tumors treated via the i.p. route (38). Drug uptake studies demonstrated an increase in ${ }^{14} \mathrm{C}$-labeled mTHPC (meta-tetrahydroxyphenylchrorin) in peritoneally disseminated tumors after i.p. administration of the drug as compared with i.v. administration (39). Taking into consideration the results of the above reports and ours, as compared with the i.v. administration route of talaporfin, the i.p. administration route increases the intratumoral concentration of talaporfin in peritoneal tumors, without increasing the concentration in other organs. Therefore, we think that the i.p. route should be the first choice for talaporfin-mediated PDT in the treatment of peritoneal tumors.

The anti-cancer effects of PDT have been theorized to be associated with not only direct singlet oxygen cytotoxicity on cancer cells but also with damage to tumor vasculature. It has been reported that responsiveness to PDT after i.v. talaporfin administration correlates with the time interval between drug administration and laser treatment, laser dose and photosensitizer levels in the plasma, but does not correlate with the photosensitizer concentration in tumor tissue, which suggests that damage to tumor blood vessels may be a primary target in talaporfin-mediated PDT $(20,37)$. Interestingly, however, there was no significant correlation between the pathological response and laser dose in i.p. talaporfin-mediated PDT in our study. In our study, the target lesions were peritoneal metastatic nodules on the omentum, where tumor blood supply is extremely poor. Previous mechanistic investigations have shown that talaporfin enters the cells via endocytosis (21). Therefore, in the case of peritoneal tumors, talaporfin might enter the tumor cells directly by endocytosis rather than by the surrounding tumor vessels. If this is true, the anti-tumor effects of i.p. talaporfinmediated PDT might be mediated by direct singlet oxygen cytotoxicity rather than by damage to tumor vasculature.

In our in vitro study, high rates of cytotoxicity were found at a treatment dose of $2 \mathrm{~J} / \mathrm{cm}^{2}$, and there were no significant differences in cell survival rates between doses ranging from 2 to $10 \mathrm{~J} / \mathrm{cm}^{2}$. Thus, a laser dose of $2 \mathrm{~J} / \mathrm{cm}^{2}$ was considered to be enough energy to consume the absorbed talaporfin in the micrometastatic nodules. In our in vivo study, we showed that a laser dose of $2 \mathrm{~J} / \mathrm{cm}^{2}$ was adequate to treat small nodules $(<1 \mathrm{~mm}$ in diameter) in i.p. talaporfin-mediated PDT.

Our study demonstrated that talaporfin accumulated in small peritoneal metastatic nodules by i.p. drug administration and that i.p. talaporfin-mediated PDT did not require a high laser dose. The pathological response rate was about $20 \%$ under optimal conditions, which is comparable to that of chemotherapy. We think that the biggest benefit of talaporfinmediated PDT is the short interval required between drug administration and light exposure. This advantage makes it possible to perform PDT immediately after surgery for gastric cancer in confirmed cases of serosa-positive cancer or in cases with positive cytology from peritoneal washings. Thus, intraperitoneally delivered talaporfin-mediated PDT seems to be a promising treatment modality for peritoneal recurrence of gastric cancer and may have a role in the prevention of peritoneal dissemination after surgery.

\section{Acknowledgments}

This research was supported in part by a Grant-in-Aid for Community Health and Medical Care from Ichou Association for Promotion of Medical Science.

\section{References}

1. Verdecchia A, Corazziari I, Gatta G, Lisi D, Faivre J and Forman D: Explaining gastric cancer survival differences among European countries. Int J Cancer 109: 737-741, 2004.

2. Maruyama K, Kaminishi M, Hayashi K, et al: Gastric cancer treated in 1991 in Japan: data analysis of nationwide registry. Gastric Cancer 9: 51-66, 2006.

3. Sun P, Xiang JB and Chen ZY: Meta-analysis of adjuvant chemotherapy after radical surgery for advanced gastric cancer. Br J Surg (In press).

4. Liu TS, Wang Y, Chen SY and Sun YH: An updated metaanalysis of adjuvant chemotherapy after curative resection for gastric cancer. Eur J Surg Oncol 34: 1208-1216, 2008.

5. Zhao SL and Fang JY: The role of postoperative adjuvant chemotherapy following curative resection for gastric cancer: a meta-analysis. Cancer Invest 26: 317-325, 2008.

6. Panzini I, Gianni L, Fattori PP, et al: Adjuvant chemotherapy in gastric cancer: a meta-analysis of randomized trials and a comparison with previous meta-analyses. Tumori 88: 21-27, 2002.

7. Mari E, Floriani I, Tinazzi A, et al: Efficacy of adjuvant chemotherapy after curative resection for gastric cancer: a metaanalysis of published randomised trials. A study of the GISCAD (Gruppo Italiano per lo Studio dei Carcinomi dell'Apparato Digerente). Ann Oncol 11: 837-843, 2000.

8. Earle CC and Maroun JA: Adjuvant chemotherapy after curative resection for gastric cancer in non-Asian patients: revisiting a meta-analysis of randomised trials. Eur J Cancer 35: 1059-1064, 1999.

9. Yan TD, Black D, Sugarbaker PH, et al: A systematic review and meta-analysis of the randomized controlled trials on adjuvant intraperitoneal chemotherapy for resectable gastric cancer. Ann Surg Oncol 14: 2702-2713, 2007.

10. Baeza MR, Giannini TO, Rivera SR, et al: Adjuvant radiochemotherapy in the treatment of completely resected, locally advanced gastric cancer. Int J Radiat Oncol Biol Phys 50: 645-650, 2001

11. Petit T, Velten M, d'Hombres A, et al: Long-term survival of 106 stage III ovarian cancer patients with minimal residual disease after second-look laparotomy and consolidation radiotherapy. Gynecol Oncol 104: 104-108, 2007.

12. Furuse K, Fukuoka M, Kato H, et al: A prospective phase II study on photodynamic therapy with photofrin II for centrally located early-stage lung cancer. The Japan Lung Cancer Photodynamic Therapy Study Group. J Clin Oncol 11: 1852-1857, 1993.

13. Mimura S, Ito Y, Nagayo T, et al: Cooperative clinical trial of photodynamic therapy with photofrin II and excimer dye laser for early gastric cancer. Lasers Surg Med 19: 168-172, 1996.

14. Prosst RL, Wolfsen HC and Gahlen J: Photodynamic therapy for esophageal diseases: a clinical update. Endoscopy 35: 1059-1068, 2003.

15. Ortner ME, Caca K, Berr F, et al: Successful photodynamic therapy for nonresectable cholangiocarcinoma: a randomized prospective study. Gastroenterology 125: 1355-1363, 2003.

16. Takita H, Mang TS, Loewen GM, et al: Operation and intracavitary photodynamic therapy for malignant pleural mesothelioma: a phase II study. Ann Thorac Surg 58: 995-998, 1994. 
17. Barr H, Krasner N, Boulos PB, Chatlani P and Bown SG: Photodynamic therapy for colorectal cancer: a quantitative pilot study. Br J Surg 77: 93-96, 1990.

18. Kobayashi W, Liu Q, Nakagawa H, et al: Photodynamic therapy with mono-L-aspartyl chlorin e6 can cause necrosis of squamous cell carcinoma of tongue: experimental study on an animal model of nude mouse. Oral Oncol 42: 46-50, 2006.

19. Grant WE, Speight PM, Hopper C and Bown SG: Photodynamic therapy: an effective, but non-selective treatment for superficial cancers of the oral cavity. Int J Cancer 71: 937-942, 1997.

20. Gomer CJ and Ferrario A: Tissue distribution and photosensitizing properties of mono-L-aspartyl chlorin e6 in a mouse tumor model. Cancer Res 50: 3985-3990, 1990.

21. Roberts WG, Smith KM, McCullough JL and Berns MW: Skin photosensitivity and photodestruction of several potential photodynamic sensitizers. Photochem Photobiol 49: 431-438, 1989.

22. Kujundzic M, Vogl TJ, Stimac D, et al: A Phase II safety and effect on time to tumor progression study of intratumoral light infusion technology using talaporfin sodium in patients with metastatic colorectal cancer. J Surg Oncol 96: 518-524, 2007.

23. Kato H, Furukawa K, Sato M, et al: Phase II clinical study of photodynamic therapy using mono-L-aspartyl chlorin e6 and diode laser for early superficial squamous cell carcinoma of the lung. Lung Cancer 42: 103-111, 2003.

24. Kaneko K, Yano M, Tsujinaka T, et al: Establishment of a visible peritoneal micrometastatic model from a gastric adenocarcinoma cell line by green fluorescent protein. Int J Oncol 16: 893-898, 2000

25. Utsuki S, Oka H, Miyajima Y, Shimizu S, Suzuki S and Fujii K: Auditory alert system for fluorescence-guided resection of gliomas. Neurol Med Chir (Tokyo) 48: 95-98, 2008.

26. Loning M, Diddens H, Kupker W, Diedrich K and Huttmann G: Laparoscopic fluorescence detection of ovarian carcinoma metastases using 5-aminolevulinic acid-induced protoporphyrin IX. Cancer 100: 1650-1656, 2004.

27. Till H, Bergmann F, Metzger R, et al: Videoscopic fluorescence diagnosis of peritoneal and thoracic metastases from human hepatoblastoma in nude rats. Surg Endosc 19: 1483-1486, 2005.

28. Collinet P, Sabban F, Cosson M, et al: Laparoscopic photodynamic diagnosis of ovarian cancer peritoneal micro metastasis: an experimental study. Photochem Photobiol 83: 647-651, 2007.
29. Major AL, Rose GS, Chapman CF, et al: In vivo fluorescence detection of ovarian cancer in the NuTu-19 epithelial ovarian cancer animal model using 5-aminolevulinic acid (ALA). Gynecol Oncol 66: 122-132, 1997.

30. Bergmann F, Stepp H, Metzger R, Rolle U, Johansson A and Till $\mathrm{H}$ : In vitro and in vivo evaluation of photodynamic techniques for the experimental treatment of human hepatoblastoma and neuroblastoma: preliminary results. Pediatr Surg Int 24: 1331-1333, 2008

31. Tan WC, Fulljames C, Stone N, et al: Photodynamic therapy using 5-aminolaevulinic acid for oesophageal adenocarcinoma associated with Barrett's metaplasia. J Photochem Photobiol B 53: 75-80, 1999.

32. Mlkvy P, Messmann H, Regula J, et al: Photodynamic therapy for gastrointestinal tumors using three photosensitizers - ALA induced PPIX, Photofrin and MTHPC. A pilot study. Neoplasma 45: 157-161, 1998.

33. Hendren SK, Hahn SM, Spitz FR, et al: Phase II trial of debulking surgery and photodynamic therapy for disseminated intraperitoneal tumors. Ann Surg Oncol 8: 65-71, 2001.

34. Friedberg JS, Mick R, Stevenson JP, et al: Phase II trial of pleural photodynamic therapy and surgery for patients with nonsmall-cell lung cancer with pleural spread. J Clin Oncol 22: 2192-2201, 2004.

35. De Laney TF, Sindelar WF, Tochner Z, et al: Phase I study of debulking surgery and photodynamic therapy for disseminated intraperitoneal tumors. Int J Radiat Oncol Biol Phys 25: 445-457, 1993.

36. Canter RJ, Mick R, Kesmodel SB, et al: Intraperitoneal photodynamic therapy causes a capillary-leak syndrome. Ann Surg Oncol 10: 514-524, 2003.

37. Ferrario A, Kessel D and Gomer CJ: Metabolic properties and photosensitizing responsiveness of mono-L-aspartyl chlorin e6 in a mouse tumor model. Cancer Res 52: 2890-2893, 1992.

38. Perry RR, Smith PD, Evans S and Pass HI: Intravenous vs intraperitoneal sensitizer: implications for intraperitoneal photodynamic therapy. Photochem Photobiol 53: 335-340, 1991.

39. Veenhuizen RB, Ruevekamp MC, Oppelaar H, Helmerhorst TJ, Kenemans P and Stewart FA: Foscan-mediated photodynamic therapy for a peritoneal-cancer model: drug distribution and efficacy studies. Int J Cancer 73: 230-235, 1997. 